\title{
Development of FEA Bioelectromagnetic Model for Male's Electromagnetic Radiation Protective Underwear ${ }^{\star}$
}

\author{
Liang Ma, Xin Zhang*, Bo-An Ying, Long Wu \\ Apparel \& Art Design College, Xi'an Polytechnic University, Xi'an 710048, China
}

\begin{abstract}
Electromagnetic radiation is the leading cause of diseases in some special environments. With the development of functional clothing design, simulation technology have been increasingly emphasized. Understanding the electromagnetic radiation protective mechanism through a FEA Bionelectromagnetic model is of great significance for male's reproductive system protection. This 3D FEA Bionelectromagnetic model includes muscle model, pelvic model, thigh bone model, bladder model, prostate model, and testis model. The influence of silver layer and bronze layer on electromagnetic field shielding within a dressed manikin exposed to $915 \mathrm{MHz}$ were investigated. Simulated results indicated that testis were protected mostly by electromagnetic radiation protective underwear and other tissues have received various degrees of damage. Increasing the fabric thickness was an effective method to reduce the electromagnetic radiation energy. Therefore, it is important to develop a bioelectromagnetic model to research the shielding effectiveness of the product. Taking the value of electric field strength, magnetic strength and SAR to evaluate influence from radiation source. The bioelectromagnetic model could help designers to forecast the performance of electromagnetic radiation protective underwear, and the results provide the basis for optimum design.
\end{abstract}

Keywords: Electromagnetic Radiation Protective Underwear; Bioelectromagnetic Model; Shielding Effectiveness

\section{Introduction}

Electromagnetic environment become more and more complicated, Medical research shows electromagnetic radiation can affect the body's thermoregultion, like atrophy of brain, cataract and lymphoid organs [1-2]. Literatures revealed that the impact of electromagnetic radiation to male's reproductive system [3]. In recent yearsmany research reported that male semen quality is declining. Some previous study on healthy men in the Chongqing area of southwest China also had indicated that semen quality was declining [4]. A variety of Experimental [5] were performed

^This work was supported by research funding from Shaanxi Province's Research Center of Apparel Engineering and Technology.

*Corresponding author.

Email address: xianzhangxin@sina.com (Xin Zhang). 
based on mice. Electromagnetic radiation of cellular telephone might have sperm toxicity in mice. A few studies on the physical method have demonstrated the ability to measure the degree of damage in human tissues from electromagnetic radiation, especially in male's reproductive system. Electromagnetic radiation protective underwear plays a key role in the field of electromagnetic radiation protection for human's health.

The FEA (Finite Element Analysis) is a numerical calculation that is widely used in engineering analysis field. From the perspective of functional analysis, finite element analysis method can be applied to solve all kinds of flow field including the material structure, mechanics research, electrical and magnetic field, fully integrated solution thermodynamics, dynamics, acoustics, super unit and fluid-structure coupling [6].

In the research of mechanism analysis, Wang Xiuchen performed some studies on the mechanism of shielding electromagnetic radiation fiber and textile, she also combined the characteristics of human body and clothing structure to explored the source of electromagnetic leakage [7-9]. Shi Xiaoning tested the shielding effectiveness of the clothing material in microwave anechoic chamber [10], and found the direction of the electromagnetic field can significantly affect the shielding effectiveness [11].

In the research of simulation analysis, in 2005, Chen Jiakui established a simplified model to simulate the shielding effectiveness when wearing clothing [12]. In 2009, Liu Chang conducted some studies on the shielding effectiveness in far-field and near-field environment of electromagnetic [13]. Su Zhentao used the foreign anatomy human body model to carry on computational numerical simulation, with a focus on the concrete influence from electromagnetic parameter. He designed a simple cube shape to replace protective clothing [14]. In 2011, Cai Mingjuan developed a human body model and simulated the distribution of electromagnetic energy, which was absorbed by the body when wearing the electromagnetic radiation protective clothing [15]. Zhang Lili used the electromagnetic simulation software Ansoft HFSS to establish a simple model that can analyze the shielding effectiveness of pregnant clothing [16]. In 2016, Zhang Shuyi adopted CST software to build a simple human body model and clothing model. He set up some probes in the human model to simulate the electric field intensity [17]. In 2017, Zhang Weiwei also took CST established five electromagnetic radiation protective clothing models to analyze the shielding effectiveness of their design. His conclusion can guide consumers to choose this kind of clothing reasonably, but he did not describe the physical experimental methods and simulation environment [18]. Sun Lifang calculated the influence of the electromagnetic absorption of tbe human anatomy model by the FDTD method [19]. Zhang Yongheng used the CATIA software to establish a female human body model and clothing model. He explored the influence from holes and joints in shielding effectiveness [20].

Using simulation technology to solve the problem of special functional clothing design based on human body will become a new trend. The main purpose of this study is to develop a bioelectromagnetic model that takes into account five parts of the human body to research the shielding effectiveness of the electromagnetic radiation protective clothing. This paper adopts the finite element method to establish a model for clothing, antenna, and environment, and a simplified local human electromagnetic model that includes the skin, fat, bones, muscles and the heart. The aim of this study is to forecast the performance of electromagnetic radiation protective clothing, and the results provide the basis for an optimal design. 


\section{Shielding Mechanism and Parameters}

\subsection{Shielding Mechanism}

Electromagnetic radiation protective clothing is aimed to block electromagnetic waves. According to the basic principle of shielding electromagnetic radiation, most of the electromagnetic waves are reflected by the garment layer, but with some of them being absorbed. Waves that go through the garment layer is harmful to the human body (Fig. 1). In daily life, people are radiated by variety of electromagnetic radiation sources, such as computer and mobile phones; thus, this paper firstly studies the electromagnetic radiation in near field.

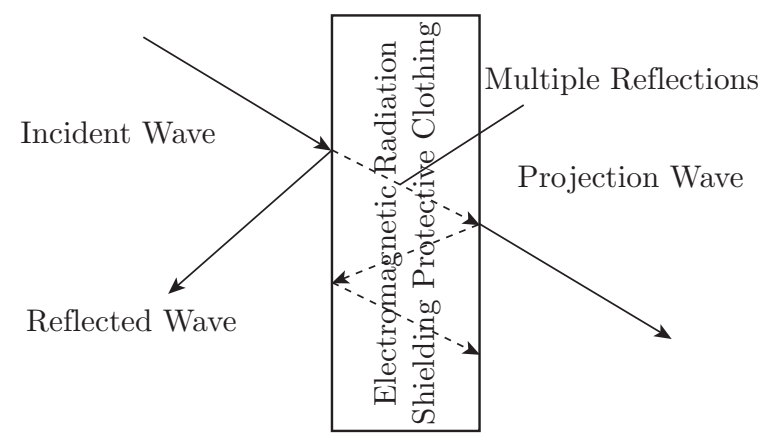

Fig. 1: Electromagnetic Radiation Shielding Mechanism

\subsection{Shielding Parameters}

Shielding effectiveness (SE) is a parameter used for shielding evaluation, which is defined as the ratio between field strength, at a given distance from the source, without the shield interposed and the field strength with the shield interposed.

(1) Shielding effectiveness of electric field-SE (E)

Electric field shielding means to weaken or eliminate the electric field strength by Electromagnetic radiation protective clothing with good conductivity. The garment structure as far as possible to reduce openings and zippers to prevent the leakage of electric field. The equation is:

$$
\mathrm{SE}=20 \lg \frac{E_{0}}{E_{1}}
$$

$\mathrm{SE}_{(\mathrm{E})}$-Shielding effectiveness of electric field, (db);

$E_{0}$ - electric field intensity without electromagnetic radiation shielding protective clothing, $(\mathrm{v} / \mathrm{m})$;

$E_{1}$ - electric field intensity with electromagnetic radiation shielding protective clothing, (v/m);

(2) Shielding effectiveness of magnetic field-SE $(\mathrm{H})$

Magnetic shielding refers to a piece of electromagnetic radiation protective clothing with good magnetic conductivity that can weaken or eliminate exposure of å the magnetic radiation from signal emitter to the human body. When the magnetic field frequency is lower, the electromagnetic 
radiation shielding clothing uses the material lower permeability, which will keep the magnetic lines from entering human body. The equation is:

$$
\mathrm{SE}=20 \lg \frac{H_{0}}{H_{1}}
$$

$\mathrm{SE}_{(\mathrm{H})}$ - shielding effectiveness of the magnetic field, (db);

$H_{0}$ - magnetic field intensity without electromagnetic shielding protective clothing, $(\mathrm{A} / \mathrm{m})$;

$H_{1}$ - magnetic field intensity with electromagnetic shielding protective clothing, $(\mathrm{A} / \mathrm{m})$;

(3) Specific absorption rate (SAR) of human body

Under the condition of external electromagnetic field, the human body will produce induction of electromagnetic field. SAR is commonly used in biometrics to characterize this physical process [21]. Specific absorption rate (SAR) is a measure of the rate at which energy is absorbed by the human body when exposed to a radio frequency (RF) electromagnetic field; although, it can also refer to absorption of other forms of energy by tissue, including ultrasound. It is defined as the power absorbed per mass of tissue and has units of watts per kilogram $(\mathrm{W} / \mathrm{kg})$.

The Equation (1) is applied to the physical quantity.

$$
a_{S A R}=10 \lg \frac{S A R_{0}}{S A R_{1}}
$$

$a_{S A R}$-attenuation factor, $(\mathrm{dB})$;

$S A R_{0}$ - specific absorption rate without electromagnetic radiation shielding protective clothing; $S A R_{1}$ - specific absorption rate with electromagnetic radiation shielding protective clothing;

\section{Electromagnetic Simulation Model}

Electromagnetic simulation model is composed of four parts, including human bioelectromagnetic model, underwear model, antenna model and environmental model. The electromagnetic simulation model coordinate system is shown in Fig. 2 and Fig. 3.

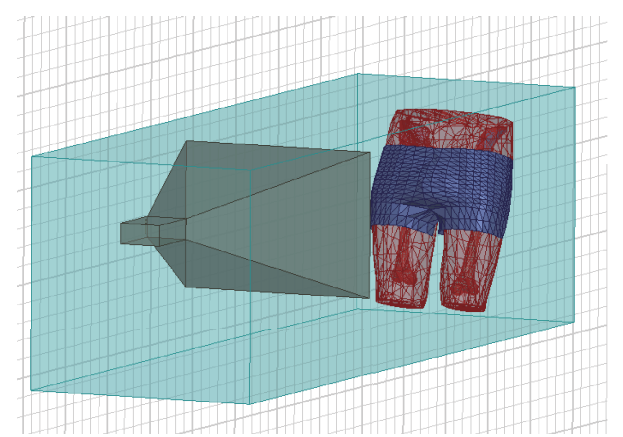

Fig. 2: Simulation mode with underwear

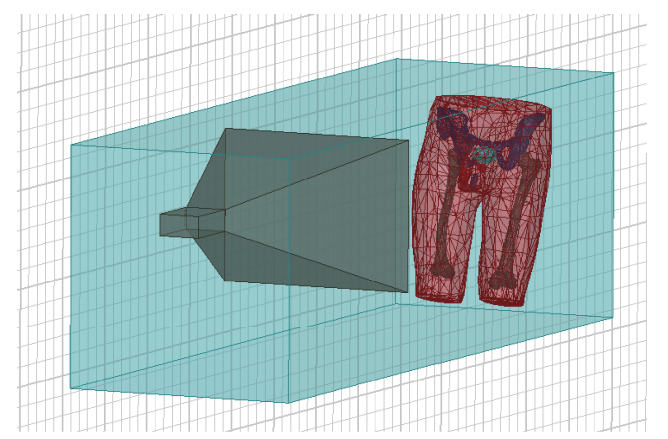

Fig. 3: Simulation mode without underwear 


\subsection{Development of 3D Male Biological Electromagnetic Model}

This 3D FEA Bionelectromagnetic model includes muscle model, pelvic model, thigh bone model, bladder model, prostate model, and testis model. The manikin geometry was built by 3D software. Due to the complex structure of the human body, the male bioelectromagnetic model is simplified when it was established, as shown in Fig. 4. According to the study of human structure, this paper established different tissues model that includes muscle model, pelvic model, thigh bone model, bladder model, prostate model, and testis model in 3D software. Assembling all parts of models as a entirety. In order to calculate the distribution of electromagnetic field by FEA method, the geometric must be mesh in FEA software. They are solid structure. On the basis of FEA model, adding biological electromagnetic properties to it. Then it become a biological electromagnetic model. The electromagnetic properties of different tissues in the human body [22] as shown in Table 1.

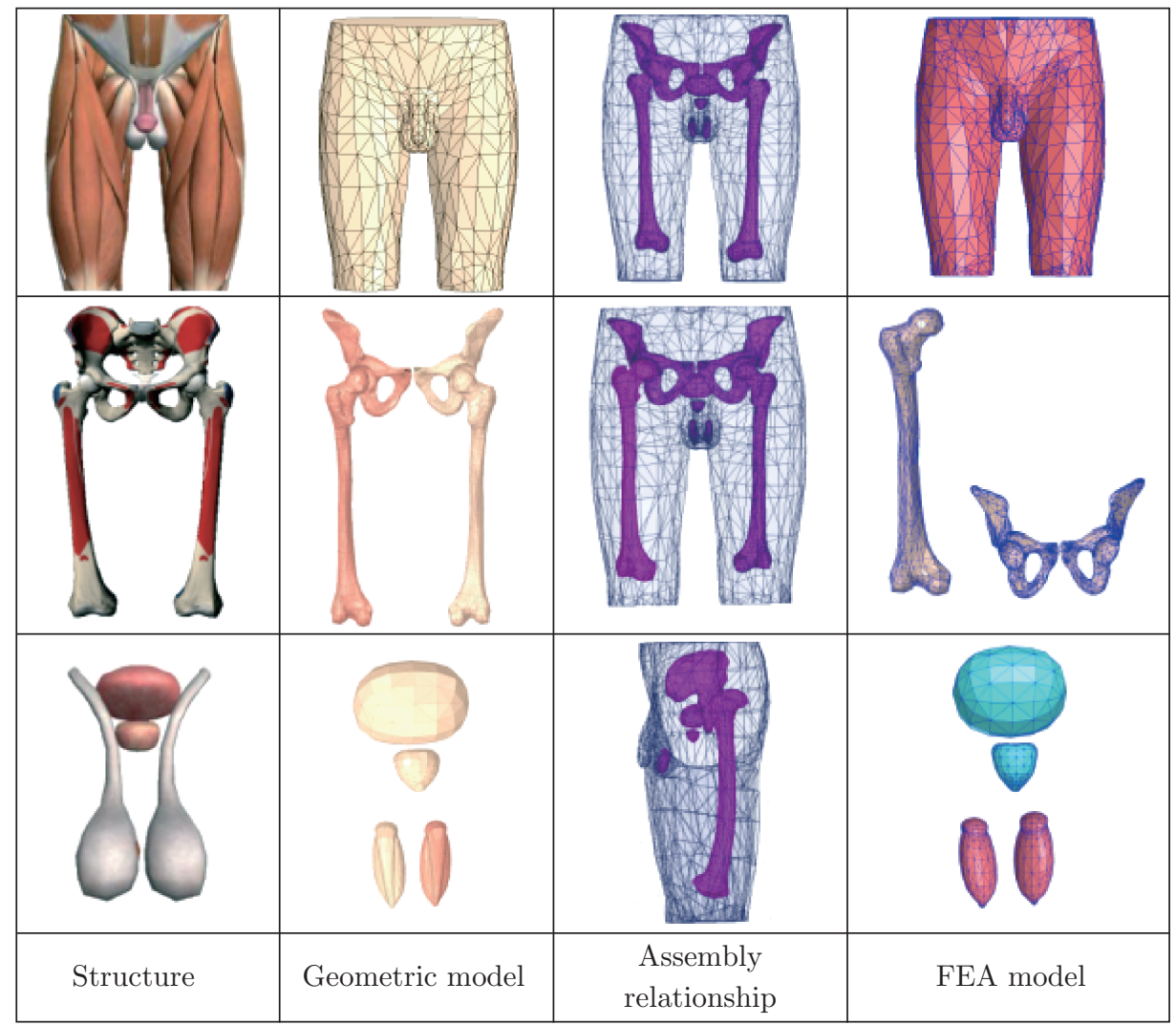

Fig. 4: Male mode

Table 1: Electromagnetic properties of human tissues

\begin{tabular}{ccc}
\hline Tissue & Relative permittivity $\varepsilon$ & Conductivity $\sigma /\left(\mathrm{S} \cdot \mathrm{m}^{-1}\right)$ \\
\hline Testis & 57.145 & 2.3859 \\
Thigh Bone & 11.23 & 0.4461 \\
Pelvic Bone & 11.23 & 0.4461 \\
Muscle & 52.412 & 1.9221 \\
Bladder (parameters of muscle) & 52.412 & 1.9221 \\
\hline
\end{tabular}




\subsection{Development of 3D Electromagnetic Shielding Protective Under- wear Model}

3D Electromagnetic Shielding Protective Underwear Model was built in 3D software. Firstly, building a plane around the human model. Secondly, establishing the front piece of underwear with the structure of underwear. Thirdly, establishing the back piece of underwear. Finally, assembling the two parts of underwear as a entirety. The process as shown in Fig. 5. Thickness of electromagnetic shielding protective underwear model is $0.5 \mathrm{~mm}$. Material of underwear is bronze, with the electromagnetic properties shown in Table 2.
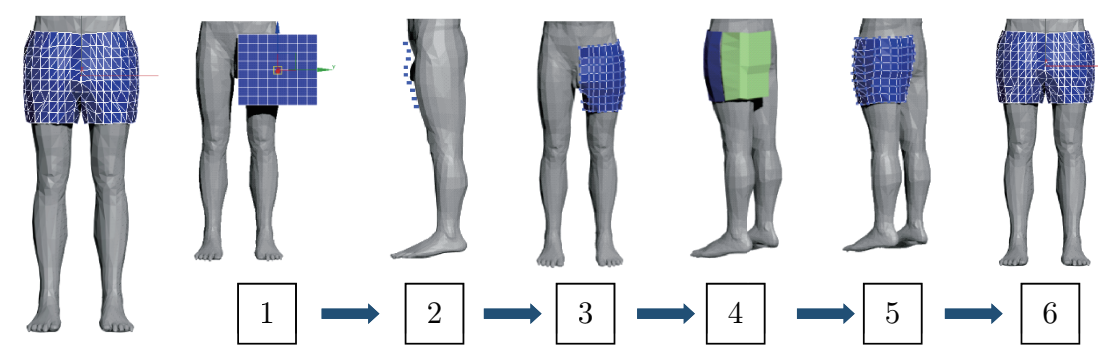

Fig. 5: Clothing model

Table 2: Electromagnetic properties of clothing

\begin{tabular}{cccc}
\hline material & Relative permittivity $\varepsilon$ & Relative permeability $\mu$ & Conductivity $\sigma /\left(\mathrm{S} \cdot \mathrm{m}^{-1}\right)$ \\
\hline Bronze & 1 & 1 & $1 * 10^{7}$ \\
\hline
\end{tabular}

\subsection{Antenna Model}

The antenna is a tool that radiates and receives electromagnetic waves. In this paper, the rectangular aperture horn antenna is used to simulate. Horn antenna is a widely used microwave antenna, and the advantages are: simple structure, bandwidth, power capacity, easy to adjust and use [23]. The geometric model mainly includes the horn model, the wave guide model and the feeder model. Signal comes from the feeder into the speaker to produce electromagnetic radiation.

\subsection{Environmental Modeling}

The environmental model is based on the conditions of simulation (Fig. 6). The space boundary condition is the radiation boundary adopted to simulate the vacuum environment. The distance

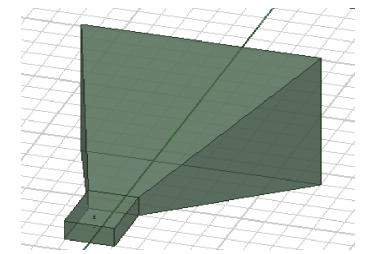

Fig. 6: Antenna model

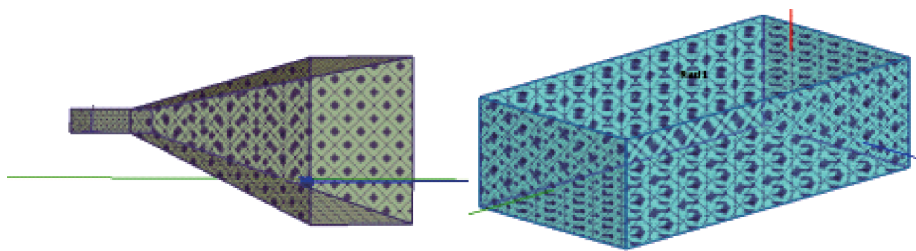

Fig. 7: Environment condition 
between the outer surface boundary and the internal model must less than $1 / 4 \lambda$. As for the antenna model, the port plane of the feeder, the port face of the antenna and the aperture plane of the antenna are ideal boundary conditions, with the rest being radiation boundaries in order to ensure its proper working status.

\section{Results and Discussion}

$915 \mathrm{MHz}$ is an usual frequency in our daily life. Radio frequency Identification (RFID) and some device are often choose this frequency. According to the standard of China, $915 \mathrm{MHz}$ is an essential point that is asked to detect. Applying the human bioelectromagnetic model, underwear model, antenna model and environmental model into simulation. Taking the value of electric field strength, magnetic field strength and SAR to evaluate influence from radiation source. The results of simulation indicate that tissues have different degree of damage. The distance between antenna and the human body is $0.5 \mathrm{~m}$. The emission frequencies are $915 \mathrm{MHz}$.

\subsection{Simulation of Electric Field Radiation}

For electric field radiation, electric field distribution of underwear layer is shown in Fig. 8. As the illustrated, underwear layer could keep electric field radiation from human body. From Fig. 9 It is clear to tell the difference of the two situation. The largest radiation area of radiation in different tissues are not same. Through the analysis of simulation results, it is illustrated that the electric field radiation strength at the position of penis is obviously larger than the others. The reason is that the wavelength of the electromagnetic and the angle of arrival in vacuum are different. According to $\lambda=\mathrm{v} / \mathrm{f}$, the largest radiation position is closer to the center. The results are shown in Fig. 9.

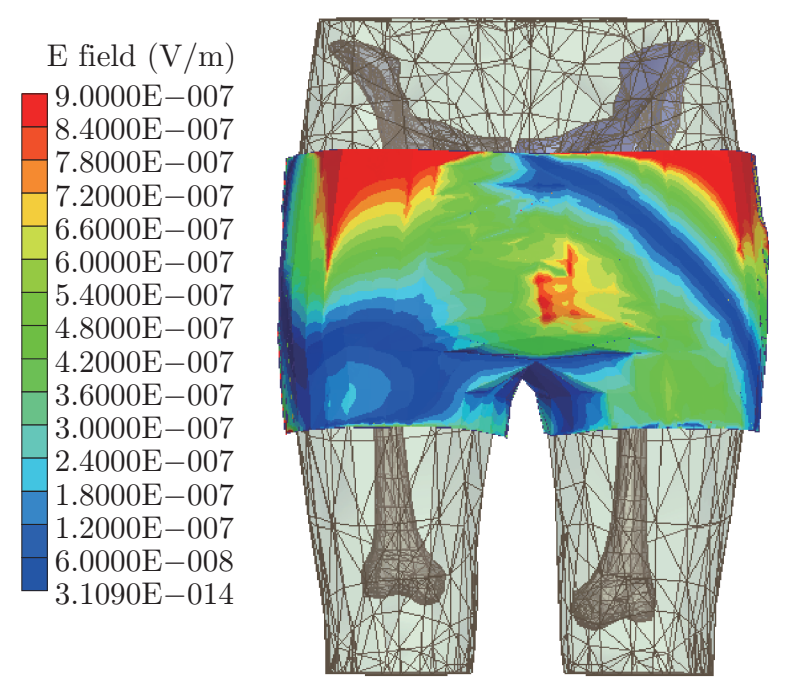

Fig. 8: Electric field radiation of underwear

In the result of simulation, horizontal cross section is aligned with antenna. From the electric field simulation results of different layer, male's reproductive system received the most radiation. It is essential to protect it. Underwear model has a good effects for reproductive system especially 

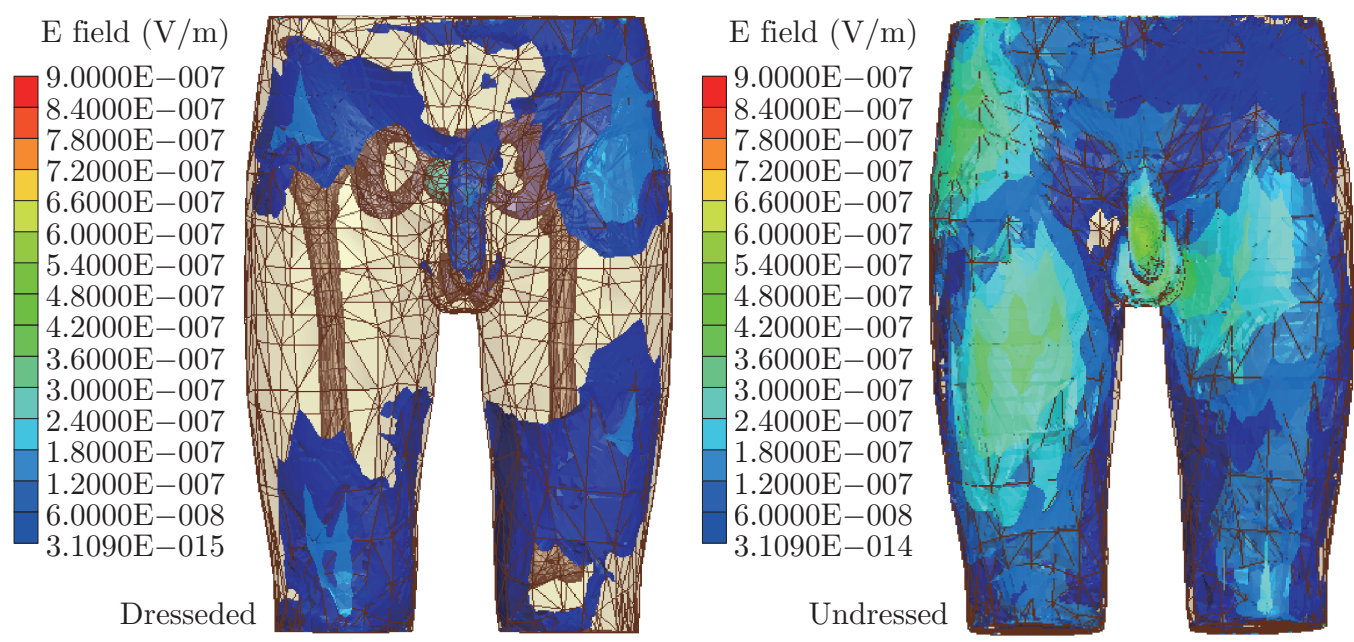

Fig. 9: Electric field radiation
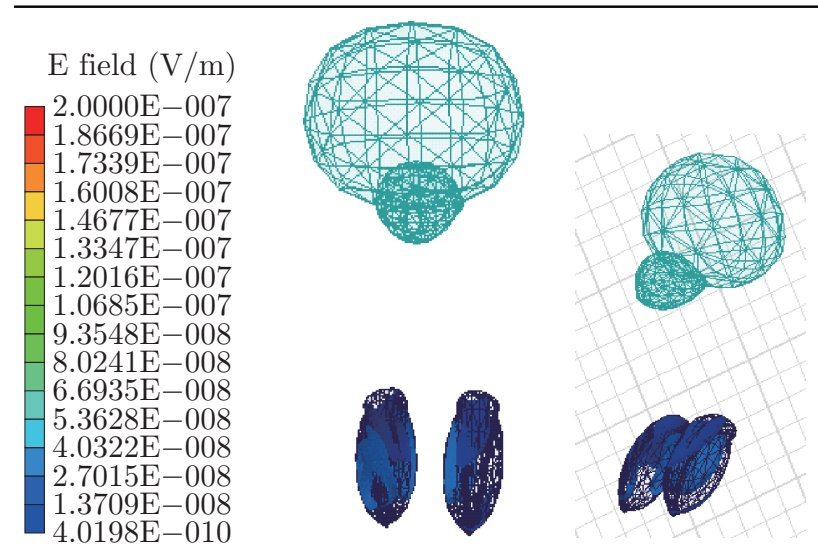

Reproductive system layer
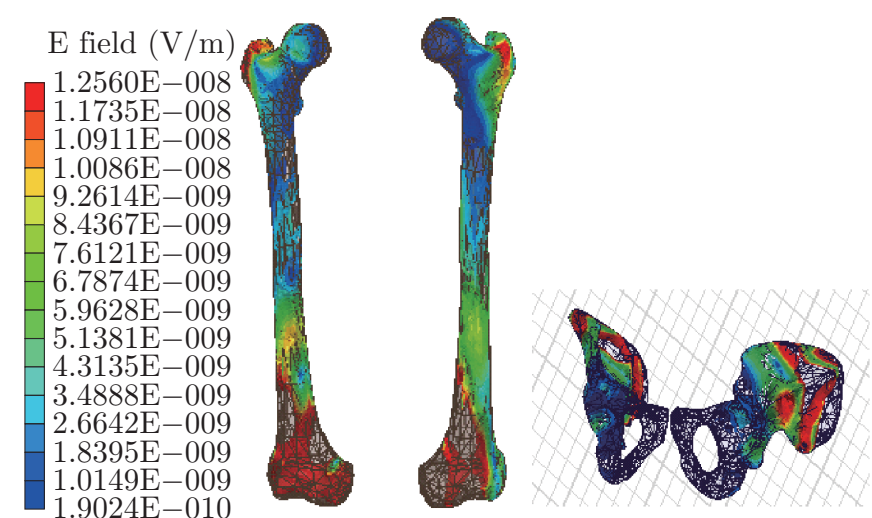

Bone layer

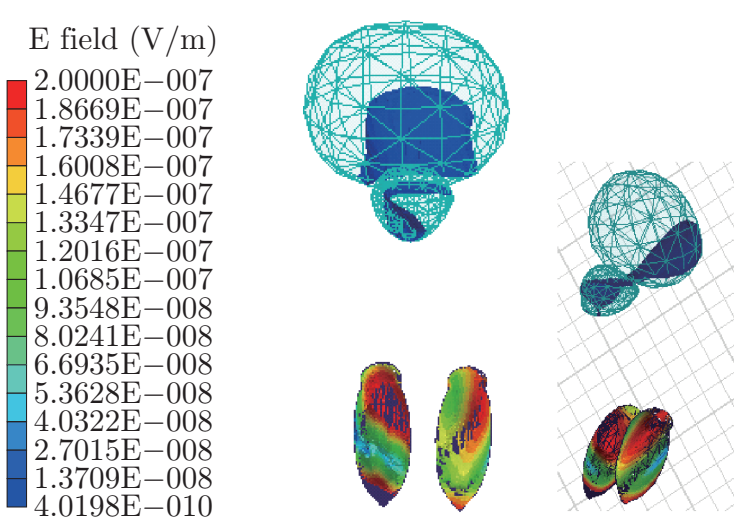

Reproductive system layer
E field $(\mathrm{V} / \mathrm{m})$

$12560 \mathrm{E}-008$

$1.1735 \mathrm{E}-008$

$1.0911 \mathrm{E}-008$

$1.0086 \mathrm{E}-008$

$9.2614 \mathrm{E}-009$

$8.4367 \mathrm{E}-009$

$7.6121 \mathrm{E}-009$

$6.7874 \mathrm{E}-009$

$5.9628 \mathrm{E}-009$

$5.1381 \mathrm{E}-009$

$4.3135 \mathrm{E}-009$

$3.4888 \mathrm{E}-009$

$2.6642 \mathrm{E}-009$

$1.8395 \mathrm{E}-009$

$1.0149 \mathrm{E}-009$

$1.9024 \mathrm{E}-010$
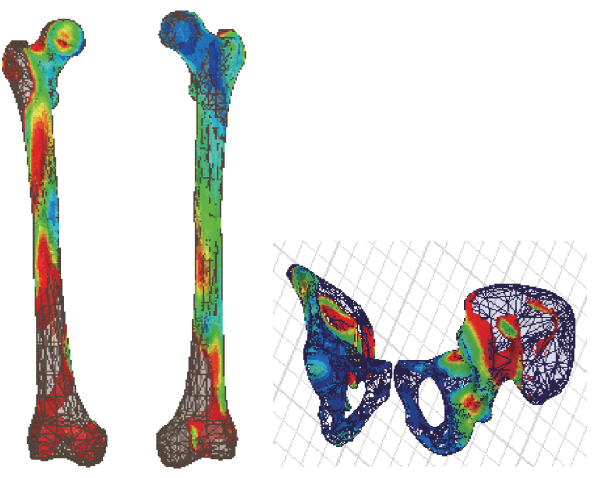

Bone layer

Fig. 10: Simulation of electric field radiation

for testis. From the distribution of electric field with $915 \mathrm{MHz}$, it is obvious that the silver clothing has a good shielding effect to the electric field and has obvious protective effect on the tissues and organs of the human body, after adding the bronze underwear layer structure. 


\subsection{Simulation of Magnetic Field Radiation}

For the magnetic field radiation, the distribution of magnetic field strength is similar to the electric field strength and emission frequency. However, power of the magnetic field radiation is smaller then electric field radiation.on Magnetic field radiation distribution of the muscle layer is as same as the electric field radiation. The largest radiation area of radiation was shown in position of penis when human is naked. Through the analysis of simulation results, it is illustrated that underwear have a good effect on human body, especially for testis. Bone layer suffered the smaller intense radiation. The results are outlined in Fig. 11-Fig. 13.

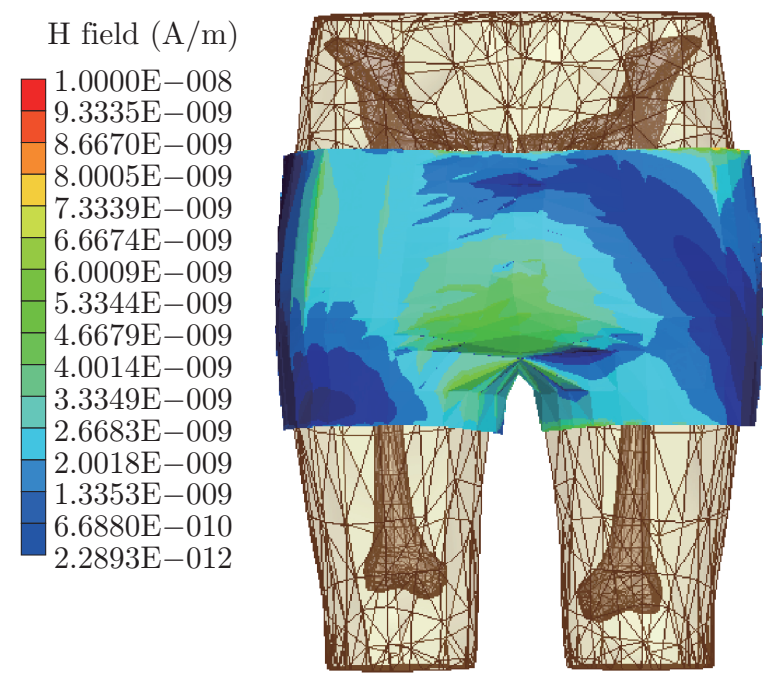

Fig. 11: Magnetic field radiation of underwear

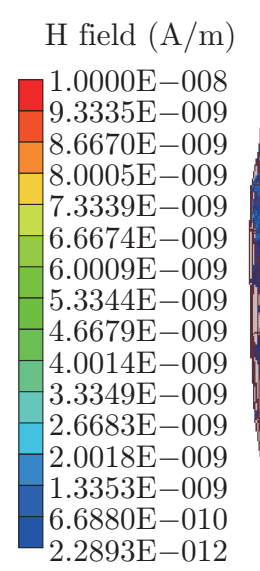

Dresseded
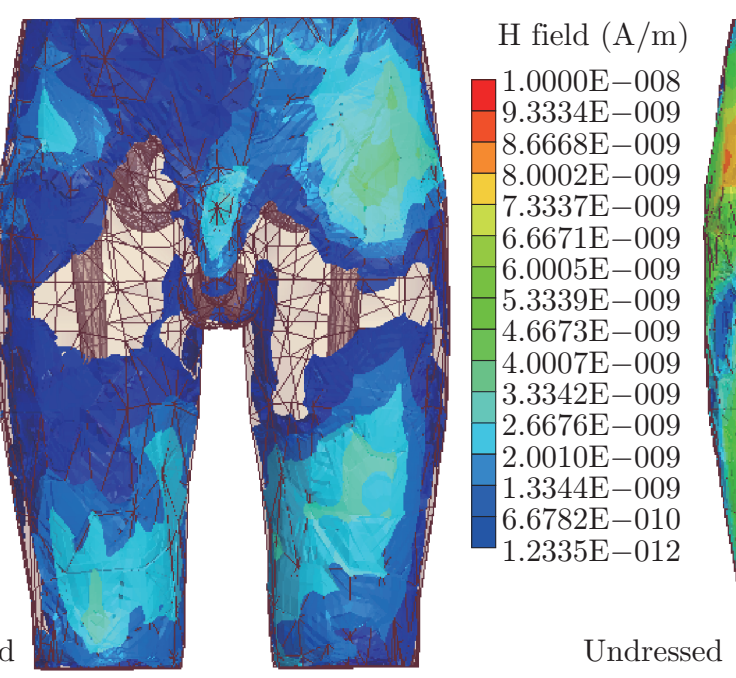

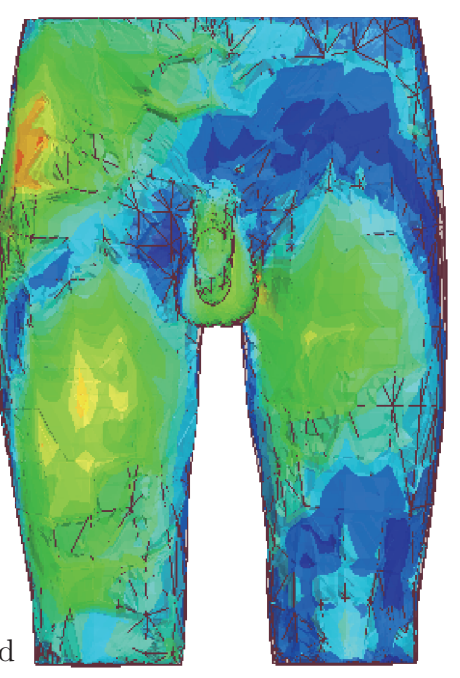

Fig. 12: Magnetic field radiation

For the distribution of SAR, The aim is calculating the distribution of the local SAR absorption. Under $915 \mathrm{MHz}$, there is a small change in the SAR value of each tissue sample. The value of SAR of the musclet layer bigger than others. Under $915 \mathrm{MHz}$, the value of SAR declined from the front to the back, but there was a significant decrease in the testis layer. The results are shown in Fig. 14 and Fig. 15. 


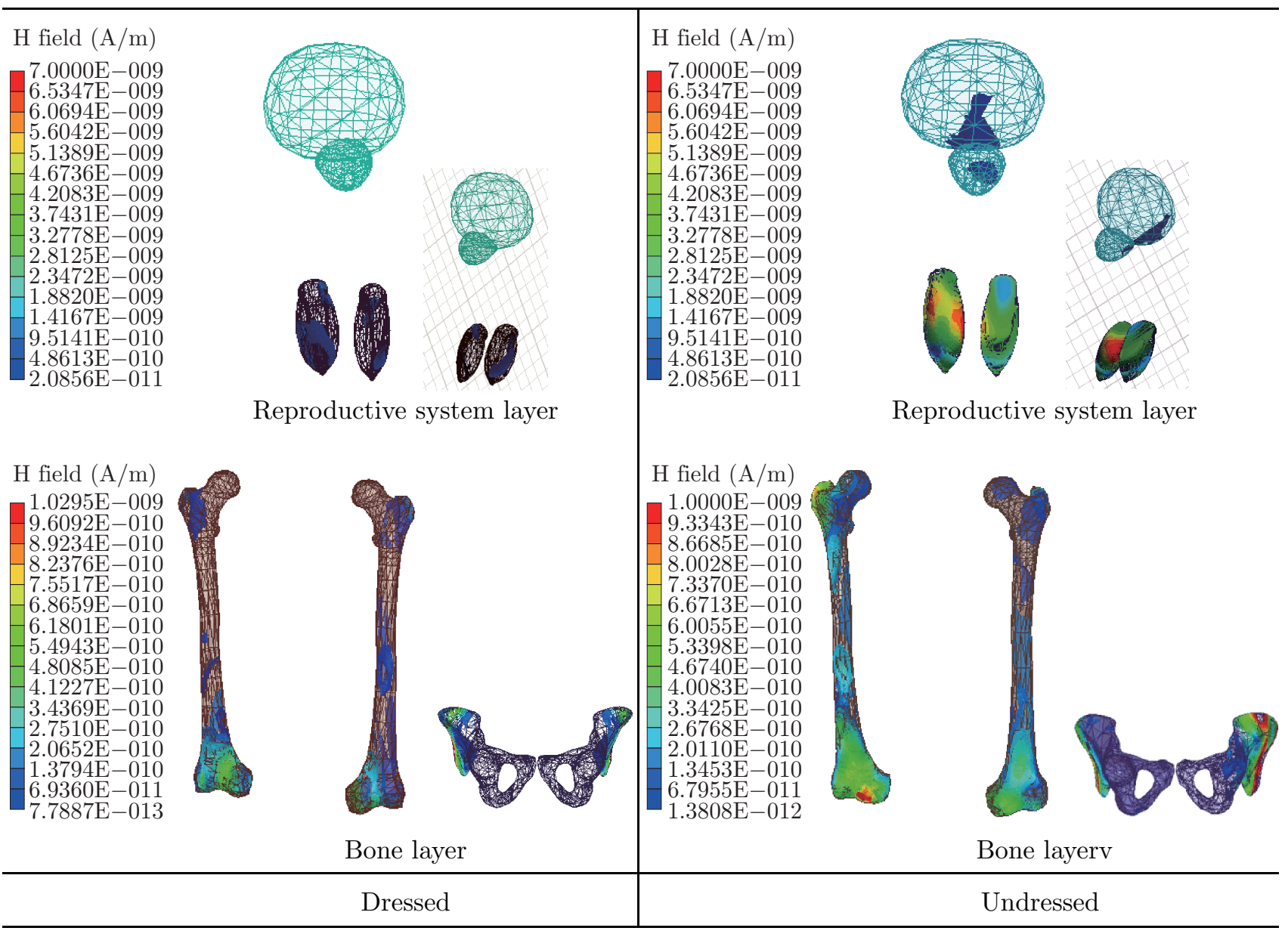

Fig. 13: Simulation of magnetic field radiation
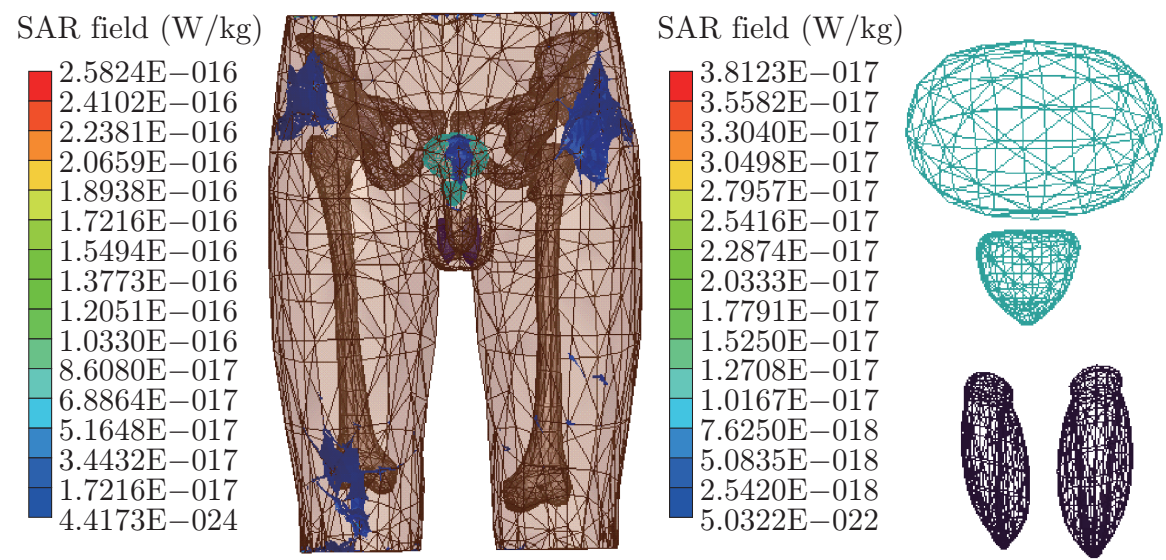

Fig. 14: Results of local SAR with underwear

\section{Conclusion}

This paper mainly studies the establishment of FEA bioelectromagnetic model for anti-electromagnetic radiation costume design, which is applied to the digital design of electromagnetic radiation shielding protective clothing. Results of the simulation indicate that tissues have re- 


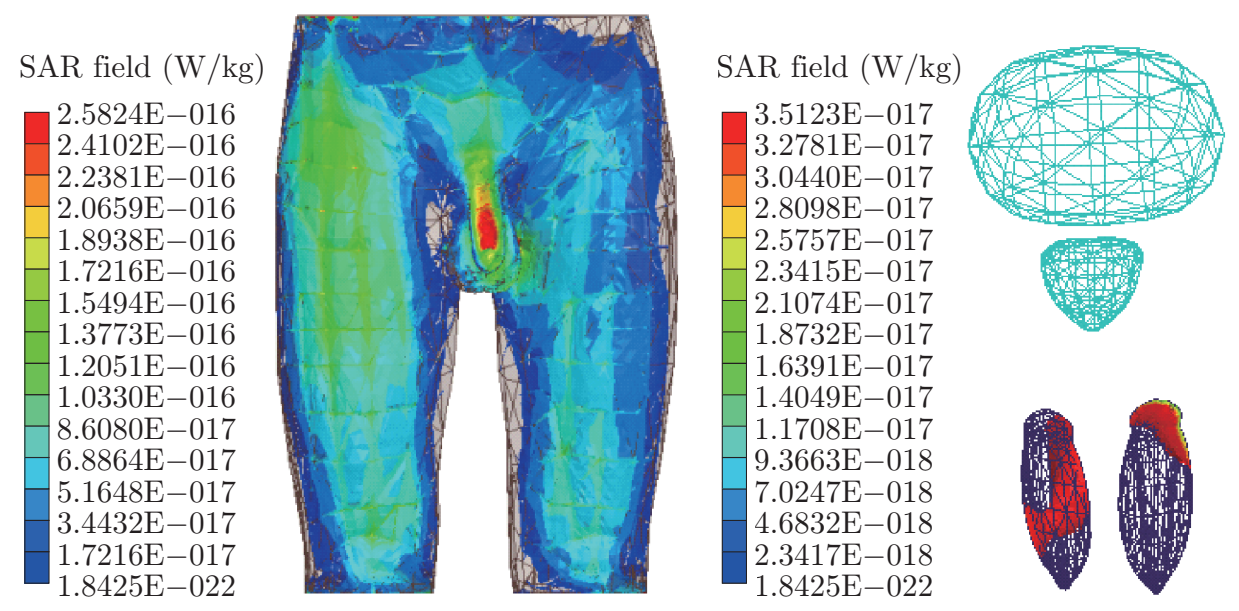

Fig. 15: Results of local SAR without underwear

ceived various degrees of damage. The bioelectromagnetic model could help designers to forecast the performance of electromagnetic radiation protective clothing, and the results could provide the basis for optimum design.

\section{Acknowledgement}

This work was supported by research funding from Shaanxi Province's Research Center of Apparel Engineering and Technology.

\section{References}

[1] Su XK. Discussion on Measuring Methods of Shielding Effectiveness of Electromagnetic Protective Clothing. Sci-Tec Innovation \& productivity, 2013; 05(95): 95-96.

[2] Wu W. Development of Anti-radiation Garment. Progress and Commentary, 2012; 19(03): 19-21.

[3] Yan B, Yuan WG. The Effects of Exposure to Electromagnetic Fields on Male Reproduction Journal of Enviroment and Occupational Medicine, 2007; 4(03): 423-425.

[4] Liu KJ. The influence of mobile phone radiation on semen quality and its biological mechanismArmy Medical University, 2016; 2-4.

[5] Cao DM, Zhou C, Zhang YZ. Study of Electromagnetic Radiation of Cellular Telephone on Sperm Damage in Mice.Industrial Health and Occupational Diseases, 2005; 6: 365-366.

[6] Xu YY. The Research of Bra Cup Fitness Based on Finite Element Analysis. Zhejiang Sci-Tech university, 2015; 12.

[7] Wang XC, Liu Z. Shielding efficiency mathematics model on the electromagnetic shielding clothing. Textile Journal, 2008; 73(04): 72-76.

[8] Wang XC, Zang X. The protection mechanism of defending electromagnetism radiates clothing. Textile Technology Progress, 2005; 26(03): 26-28.

[9] Wang XC, Zhang X. Analysis of significant factors of influence shielding efficiency on electromagnetic shielding clothing. Journal of Xi'an University of Engineering Science and Technology, 2006; 19(2): 397-401. 
[10] Shi XN. Measurement and influence factors for shielding effectiveness of material based on shielding enclosures method. North China Electric Power University, 2015; 32-35.

[11] Xiao H, Shi MW, Chao S. Influence of metal yarns arrangement mode on shielding effectiveness. Textile Journal, 2015; 36(12): 25-31.

[12] Chen JK. Research on the Test Method for Measuring the Shielding Effectiveness of Electromagnetic Shielding Clothing. Beijing: Beijing University of Posts and Telecommunications, 2017: $17-19$.

[13] Liu C. Research on the Test Method for Measuring the Shielding Effectiveness of Electromagnetic Shielding Clothing. Beijing University of Posts and Telecommunications, 2009: 25-27.

[14] Sun ZT. Shielding Effectiveness Evaluation of EM Shielding material. Radiation Protection, 2009; 04(29): 23-26.

[15] Cai MJ. Research on Electromagnetic Simulation of Characteristics of Electromagnetic Shield Suit to Body. National University of Defense Technology, 2011: 170-172.

[16] Zhang LL, Chen Y. Testing and simulation of electromagnetic shielding effects of electromagnetic protective clothing for pregnant women. Textile Journal, 2011; 32(10): 108-122.

[17] Zhang ST. Research On Shielding Effectiveness Testing Method Of Shielding Suits. Southeast University, 2016: 20-28.

[18] Zhang WW. Shielding Effectiveness Evaluation of Electromagnetic Shielding Clothing. Sci-Tec Innovation \& productivity, 2017; 06(106): 107-113.

[19] Sun LF. Simulation of shield effectiveness of protective suits. East China Normal University, 2017: $28-30$.

[20] Zhang YH. Research the Shielding Effectiveness of Electromagnetic Shilding Garments on the Three-Dimension Simulation. Zhong yuan University of techonlogy, 2017: 60-66.

[21] Jing H, Xu Z P. Electromagnetic Radiation and Human Health. Chinese Journal of Industrial Hygiene and Occupational Diseases, 2002; 20(01): 241-242.

[22] Deng PY. The numerical simulation of electric field distribution in human body exposed to electromagnetic plane waves between $0.9 \mathrm{GHz}$ to $10 \mathrm{GHz}$. East China Normal University, 2012: 14-16.

[23] Li M, Li MY. HFSS antenna design. Electronic Industry Press, 2011: 280-320.

[24] Xie CF, Yao KJ. Electromagnetic field and electromagnetic wave. Higher Education Press, 2006: $10-45$. 